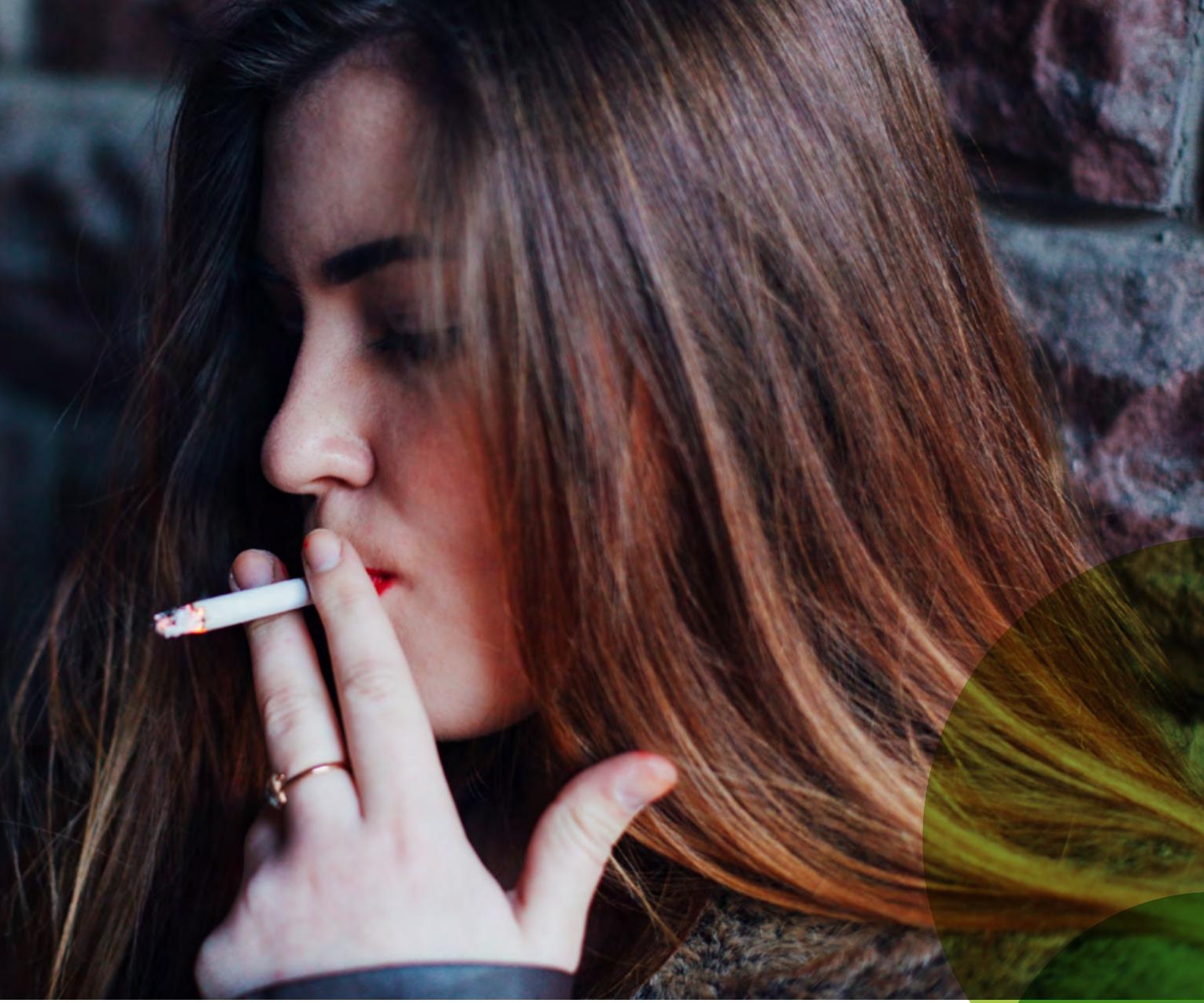

\title{
Do brain function abnormalities
to substance use, or vice versa?
}

By Dr. Jessica Edwards

New research has, for the first time, investigated the direction of links between brain function and substance use throughout adolescence. Jungmeen KimSpoon and colleagues studied 167 adolescents who were assessed annually for four years from 13-14 years old. The researchers assessed risk-related brain function by measuring blood-oxygen-level-dependent responses in the insula (a brain region involved in processing risk information) during a lottery choice task. They assessed substance use by asking about cigarette, alcohol, and marijuana use. Additionally, they assessed cognitive control, a possible moderator, by evaluating behavioural performance during the Multi-Source Interference Task (which required participants to indicate the identity of a unique digit within a sequence despite this digit not matching its position on the button-press).

Kim-Spoon and colleagues found that greater substance use predicted subsequent increased insula activation during risk processing, and this relationship was particularly strong for adolescents with low cognitive control. Cognitive control might, therefore, moderate neurological changes after substance use. In contrast, insula activation was not significantly linked with subsequent substance use. Although the researchers acknowledge important limitations to their study, they hypothesize that cognitive control might serve as an important target in preventing and treating substance use in adolescents. 